\title{
Gaze cuing and affective judgments of objects: I like what you look at
}

\author{
ANDREW P. BAYLISS, MATTHEW A. PAUL, PETER R. CANNON, and STEVEN P. TIPPER \\ University of Wales, Bangor, Wales
}

\begin{abstract}
When we see another person look somewhere, we automatically attend to the same location in space. This joint attention emerges early in life and has a great impact on social interactions in development and in everyday adult life. The direction of another's gaze indicates what object is of current interest, which may be the target for a subsequent action. In this study, we found that objects that are looked at by other people are liked more than objects that do not receive the attention of other people (Experiment 1). This suggests that observing averted gaze can have an impact on the affective appraisals of objects in the environment. This liking effect was absent when an arrow was used to cue attention (Experiment 2). This underlines the importance of other people's interactions with objects for generating our own impressions of such stimuli in the world.
\end{abstract}

Analyzing the gaze of other people is a behavior that is present virtually from birth (e.g., Moore \& Dunham, 1995; Vecera \& Johnson, 1995). The language of the eyes is a complex one, communicating a current emotional state highly efficiently to other members of the social group (Argyle \& Cook, 1976; Emery, 2000). Furthermore, the direction of gaze indicates the current attentional state. That is, the direction of gaze indicates what object a conspecific is currently interested in. Observing a shift in eye gaze results in a corresponding fast and obligatory shift of attention in the observer (e.g., Friesen \& Kingstone, 1998; Langton \& Bruce, 1999), to align his or her own attention system with that of the other person - that is, joint attention.

A great deal of research has already been informative about the nature of gaze-evoked shifts of attention (e.g., Bayliss, di Pellegrino, \& Tipper, 2004; Friesen \& Kingstone, 2003; Frischen \& Tipper, 2004). However, it is possible that observing another individual's gaze shift might not just activate the observer's attention system into the same state, where the observer shifts attention toward the same location, but might also trigger simulation of other processes associated with gaze direction. For example, when one looks at an object, it not only becomes the focus of one's attention, but also reflects preference formation, as gaze tends to settle on objects that one decides that one likes (Shimojo, Simion, Shimojo, \& Scheier, 2003). Indeed, 4-year-olds understand this link between gaze direction and object preference (Baron-Cohen, Campbell, Karmiloff-Smith, Grant, \& Walker, 1995). Thus, when one

This work was supported by an Economic and Social Research Council (ESRC-UK) grant awarded to S.P.T. and by an ESRC Postdoctoral Fellowship award to A.P.B. Correspondence concerning this article should be addressed to A. P. Bayliss, School of Psychology, University of Wales, Brigantia Building, Penrallt Road, Bangor, Gwynedd LL57 2AS, Wales (e-mail: a.bayliss@bangor.ac.uk). looks toward an object, one tends to like it more than an object that one looks away from. The critical question we ask is whether similar emotional responses are activated in an individual who observes another person look toward or away from objects: Do participants prefer objects that are looked at by other people (Experiment 1)? One might expect such an effect to be absent when attention is cued by an arrow (Experiment 2).

In this study, participants saw a face in the center of the screen that could look left or right. This face was irrelevant to the participant's task of object categorization, so any effects due to gaze direction would be automatically evoked and be nonstrategic. The critical manipulation was that for each participant, there were two versions of 36 household objects, identical except for color. One version of each object would be looked at by the face every time it appeared, whereas the face would look away from the other (differently colored) object every time it appeared. It was predicted that the participants would like the lookedat object more than the objects never looked at. This finding would lend support to the idea that the gaze behavior of others can influence the way we evaluate visual stimuli in the environment.

\section{EXPERIMENT 1}

\section{Method}

Participants. Twenty-four adults ( 5 of them male) volunteered for this study. The mean age was 20.8 years ( $S D=1.31$ years), and all had normal or corrected-to-normal vision. The participants were recruited from the School of Psychology's undergraduate participant panel, gave informed consent, and received course credit for participation.

Stimuli. The stimuli were presented approximately $60 \mathrm{~cm}$ away from the participant. Forty unfamiliar faces with neutral or moderately positive facial expressions were used as cue stimuli. These faces were framed in windows measuring $9.8 \times 14.0 \mathrm{~cm}$. The faces themselves varied between 6.3 and $7.6 \mathrm{~cm}$ in width and between 8.5 and $11.5 \mathrm{~cm}$ in height. Three versions of each face were produced, 
one with gaze straight ahead, one with the pupils averted leftward, and another with the pupils averted rightward. The eye regions were approximately $0.5 \mathrm{~cm}$ high and varied between 4.0 and $5.0 \mathrm{~cm}$ in width. The pupils measured approximately $0.5 \times 0.5 \mathrm{~cm}$. Which face produced the cue on each trial was randomized. The fixation cross, which preceded each trial, measured $0.8 \times 0.8 \mathrm{~cm}$.

The target stimuli consisted of 36 household items, 18 of which could generally be found in the garage (household tools-e.g., a screwdriver, a pair of pliers, an axe, and a saw). The other 18 were kitchen items (e.g., a kettle, cutlery, a mug, and a saucepan). These stimuli could appear in one of four colors: red, blue, green, or yellow. The hue of the main section of each object was manipulated in Adobe Photoshop. Thus, there were a total of 144 possible target stimuli that could appear in their original orientation or flipped about the vertical axis. The targets were presented so that the centers of the targets were $14 \mathrm{~cm}$ from the center of the screen. The targets varied between 3.6 and $10.5 \mathrm{~cm}$ in width and between 1.5 and $7.4 \mathrm{~cm}$ in height. For the recording of preference ratings of the stimuli, a chart was presented, with the message "How much did you like that object?" at the top of the screen and with a column of numbers from 9 to 1 , headed by the words "Like very much" at the top and the words "Don't like at all" at the bottom of the screen.

Design. The cuing factor was whether the target was looked at (congruent), or looked away from (incongruent). The manner in which the targets (kitchen/garage items) were presented to each participant was tightly controlled. Although there were four colors that each object could appear in, each participant would see only two examples of each stimulus. To compensate for any problems for which the participants might simply rate objects in their favorite color more highly, we randomized which color was selected for each target, for each participant. Furthermore, which exemplar of each colored target would serve as the consistently congruent item and the consistently incongruent item across six exposures was also randomly assigned.

Procedure. In the standard cuing trials of Blocks 1 to 5 , the participants, with their head position supported by a chinrest, were told to fixate the fixation cross, to refrain from eye movements, to ignore the uninformative gaze cue, and to respond to the target as quickly as possible. At the start of each trial, a fixation cross would appear for $600 \mathrm{msec}$. Then a face would appear, with eyes gazing straight ahead, for $1,500 \mathrm{msec}$; then, $500 \mathrm{msec}$ before target onset, the eyes would move to the left or the right. After $2,500 \mathrm{msec}$, or after response, the screen would go blank for $1,500 \mathrm{msec}$ before the next trial (see Figure 1A). At response, a tone was sounded to give feedback on performance (a bell for correct and a buzzer for incorrect/timeout). The participants used the " $h$ " key and the space bar to respond to the category of the target object. Whether " $h$ " corresponded to garage or kitchen items was counterbalanced between participants. After 16 practice trials (using the same cue faces but

A
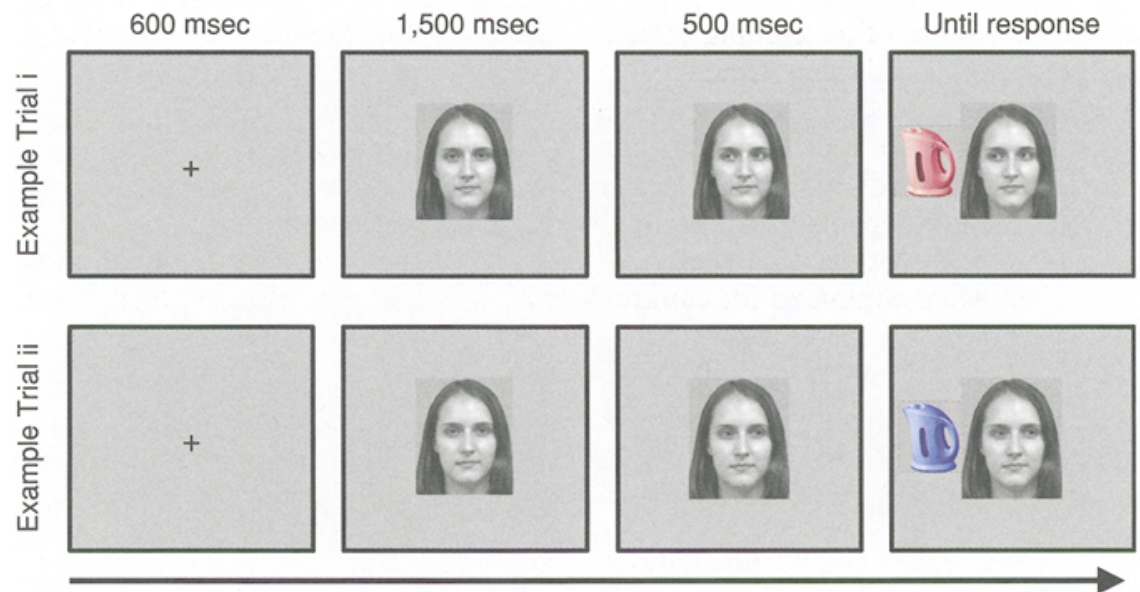

Time

B

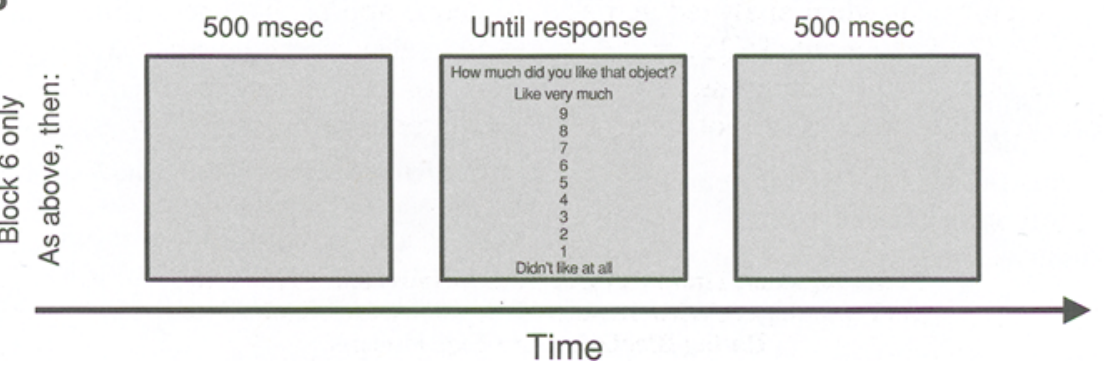

Figure 1. (A) Illustration of the time course of two sample trials. At the top, the red kettle is congruent; at the bottom, the blue version of this item is incongruent. Throughout the experiment, this cuing relationship was maintained. Thus, in this example, the red kettle was always gazed at in each of the six exposures. The cue face could be one of 40 , and the colors of the target items could also be green or yellow; any of these colors could be the congruent or the incongruent item for different participants. (B) Illustration of the time course of the subsequent ratings screen appearing after response in the final (sixth) block only. In Experiment 2 , a simple arrow replaced the face. 
different target objects), the participants completed five blocks of 72 trials, in which each target was viewed once in each block.

In the final (sixth) rating block, the procedure changed. The participants were now informed that following their target categorization response, a blank screen would be presented $(500 \mathrm{msec})$, followed by a ratings screen (see Figure 1B). They were required to rate the item they had just responded to, so that higher scores were given to objects that they preferred. They were told to verbally state a number from 1 to 9 , to be recorded by the experimenter. After their liking rating, another blank screen $(500 \mathrm{msec})$ preceded the next trial. In total, therefore, the participants completed 432 trials of the gaze-cuing procedure, being exposed six times to 36 congruent targets and six times to 36 incongruent targets. They rated each of these 72 targets once in the final (sixth) block.

\section{Results}

Gaze cuing. Accuracy in categorizing the targets was $95.8 \%$. For errors, the effect of cuing was nonsignificant in the standard blocks $[t(23)=-0.932, p=.36]$ and the rating block $[t(23)=0.632, p=.53]$. Analysis of median reaction times (RTs) revealed the standard gaze-cuing effect, with RTs being shorter to congruent than to incongruent targets (see Table 1). This was significant in the standard blocks $[t(23)=2.211, p=.019$, one-tailed $]$ and marginal in the ratings block $[t(23)=1.701, p=.051$, one-tailed].

Object ratings. The replication of gaze-cuing effects was of secondary importance in this experiment. The central issue was whether this gaze shift would produce a higher rating for objects that were looked at than for objects that were not looked at. Immediately after the sixth exposure to a target item, the participants rated the item from 1 to 9 . Ratings from items that were correctly responded to in the final block were compared for congruent and incongruent items. Crucially, there was a significant cuing effect $[t(23)=4.64, p<.001 ; r=.49]$, with the items that were consistently looked at throughout the experiment being rated more preferable $(M=4.74, S D=$ 0.73 , range $=3.15$ ) than were the items consistently looked away from $(M=4.59, S D=0.78$, range $=3.48)$. Although the overall difference was small, the analysis demonstrated that the effect was highly reliable, being shown by 22 of 24 participants (see Figures 2 and 3 ). The liking effect was also significant when analyzed across materials, rather than across participants $[t(35)=2.63$, $p=.013 ; r=.30]$. Interestingly, the magnitude of a participant's liking effect evoked by the cues did not correlate with the magnitude of the participant's cuing effect $(r=$ $-.156, n=24, p=.46$ ).

\section{Discussion}

In this experiment, we investigated whether simply observing someone look toward an object that requires a response results in a more positive affective appraisal of that object than of objects that are not looked at. Along with the standard gaze-cuing effect, we found that visual objects that consistently appeared in congruent locations (i.e., looked at six times out of six appearances) were rated as more likable than objects appearing in incongruent locations (i.e., looked away from). This finding is a further demonstration of the importance of other people's behavior in determining our beliefs and preferences. In this case, subtle behaviors such as another person's gaze shifts, even when they are not relevant to our goals, can have significant influences on what we like.

There are two plausible reasons for the liking effect. First, the shift of attention in the direction of gaze facilitates RTs to congruent items. This perceptual fluency is associated with the congruent items; hence, they attract a more positive emotional evaluation than do incongruent targets (Reber, Winkielman, \& Schwarz, 1998). However, if the shift of attention per se were associated with the evaluation of the item, one would predict that the stronger a participant's shift of attention, the larger the difference between the ratings of congruent and incongruent items would be. However, as was noted above, there was no hint of such a correlation.

The second possible explanation is that the gaze cue acts as a social reinforcer for items in the environment. That is, our preferences for objects are influenced by the preferences that other people display. Since gaze direction is an excellent indicator of another person's interest (Baron-Cohen et al., 1995; Shimojo et al., 2003), it is very likely that the objects that they inspect are objects that they find attractive and the objects that people avert gaze from are unattractive. This alludes to the distinction between approach and avoid emotional responses to stimuli (see Davidson \& Irwin, 1999, for a review). If we observe someone approach/avoid a stimulus, it will serve us well to generate a matching emotional tag to that stimulus. This is one of the primary reasons that an eye direction detector might have evolved in large social groups-to benefit

Table 1

Mean Reaction Times (RTs, in Milliseconds) and Error Rates (As Percentages), With Standard Deviations, for Standard and Rating Blocks in Both Experiments

\begin{tabular}{|c|c|c|c|c|c|c|c|c|c|}
\hline & \multirow[b]{3}{*}{ Condition } & \multicolumn{4}{|c|}{ Standard Blocks } & \multicolumn{4}{|c|}{ Rating Block } \\
\hline & & \multicolumn{2}{|c|}{ RT } & \multicolumn{2}{|c|}{ Errors } & \multicolumn{2}{|c|}{ RT } & \multicolumn{2}{|c|}{ Errors } \\
\hline & & $M$ & $S D$ & $M$ & $S D$ & $M$ & $S D$ & $M$ & $S D$ \\
\hline Experiment 1 & $\begin{array}{l}\text { Incongruent } \\
\text { Congruent }\end{array}$ & $\begin{array}{l}672 \\
663\end{array}$ & $\begin{array}{l}92 \\
87\end{array}$ & $\begin{array}{l}4.31 \\
4.75\end{array}$ & $\begin{array}{l}3.7 \\
3.1\end{array}$ & $\begin{array}{l}714 \\
692\end{array}$ & $\begin{array}{l}127 \\
114\end{array}$ & $\begin{array}{l}2.78 \\
2.20\end{array}$ & $\begin{array}{l}3.7 \\
2.6\end{array}$ \\
\hline Experiment 2 & Incongruent & 646 & 67 & 6.04 & 3.7 & 698 & 108 & 3.01 & 3.3 \\
\hline & Congruent & 630 & 61 & 5.39 & 3.4 & 683 & 101 & 4.05 & 3.7 \\
\hline
\end{tabular}




\section{Experiment 1-Gaze Cue Experiment 2-Arrow Cue}
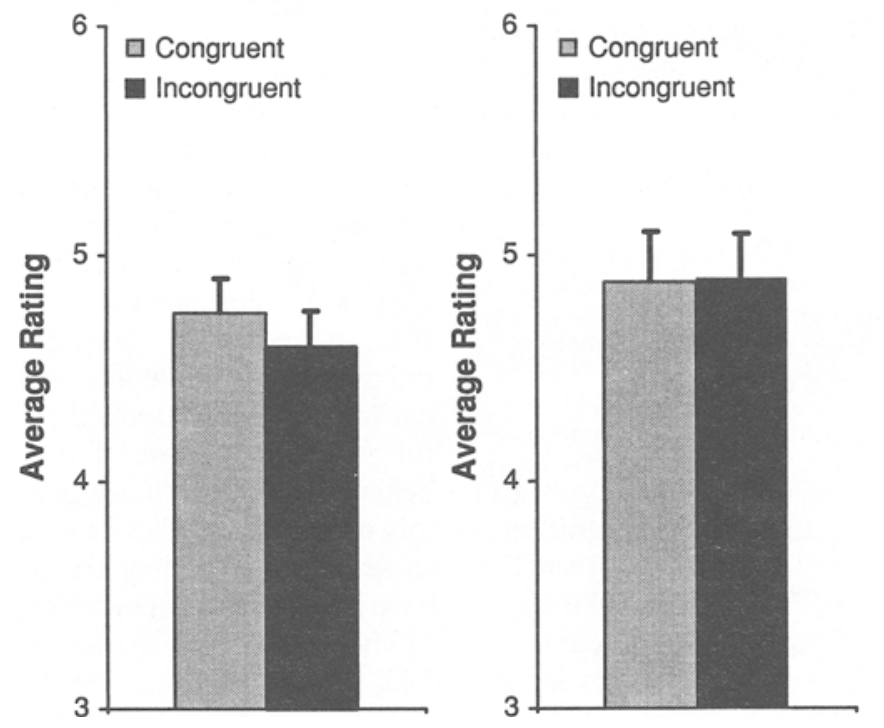

Figure 2. Bar chart illustrating the average rating (between 1 and 9) assigned by the participants to congruent or incongruent garage and kitchen items (with standard error bars), for both the experiments. Higher ratings indicate that the participants liked the objects more.

from other people's knowledge and behavior by efficiently interpreting their behavior (Baron-Cohen, 1995).

A second experiment was therefore conducted in order to establish which of these two hypotheses best explains the results of Experiment 1. By simply using an arrow cue, instead of a gaze cue, the data would lend support to one or the other hypothesis. If an arrow cue were to produce attentional cuing and a liking effect, it would suggest that attention boosts appraisal of objects, whether attention is shifted by gaze or otherwise. If, however, an arrow cue were to produce cuing but no liking effect, we would have confirmed that in this paradigm, objects that other people look at receive more positive appraisals than do ones that are not looked at, independently of attention.

\section{EXPERIMENT 2}

Like gaze cues, arrows shift attention, even when participants are instructed to ignore them (e.g., Shepherd, Findlay, \& Hockey, 1986; Tipples, 2002). The cuing effects elicited by arrows are very similar to those produced by gaze cues, in terms of their time course, magnitude, and sensitivity
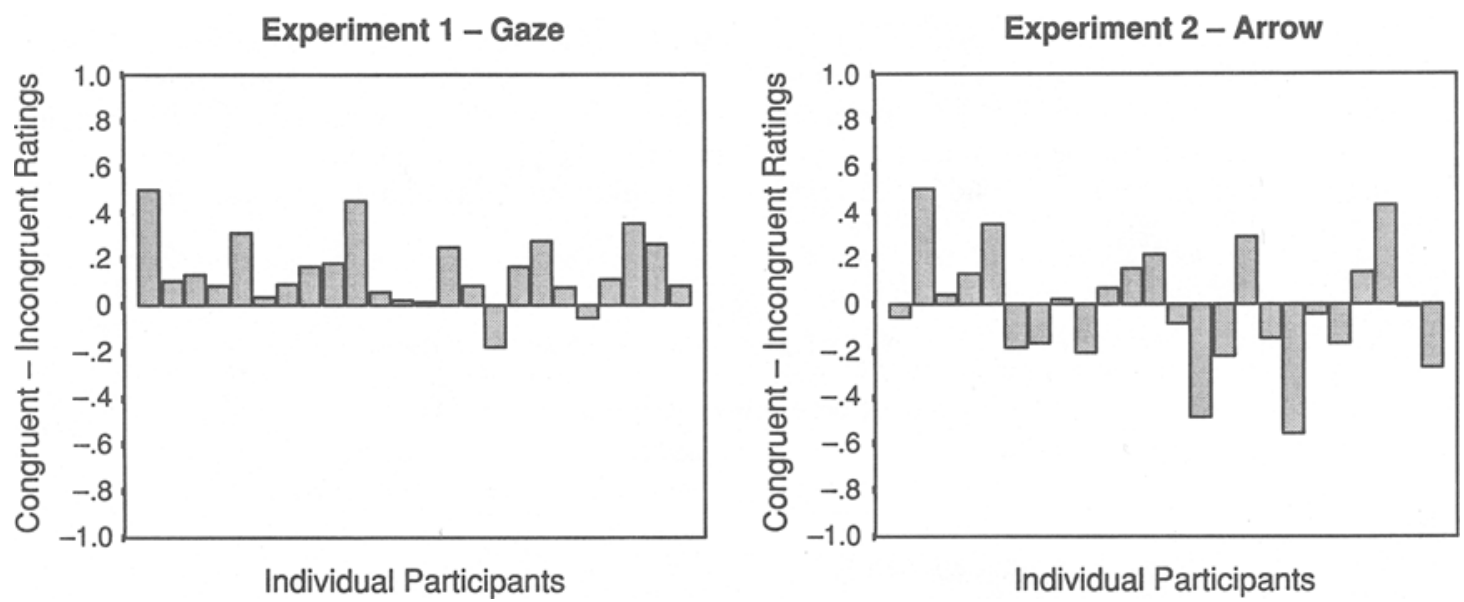

Figure 3. Bar charts for each experiment, illustrating the rating biases shown by individual participants (in the order in which they were recruited). Hence, bars above the $x$-axis illustrate that a participant rated congruent items higher than incongruent items. 
to individual differences (e.g., females show more cuing following gaze and arrow cues than males do; Bayliss, di Pellegrino, \& Tipper, 2005). In general, therefore, the attention shift in this experiment would be similar to that in the previous experiment. The critical measure again, however, is whether there is a difference between congruent and incongruent objects in participant's ratings of liking.

\section{Method}

The method for this experiment was identical to that in Experiment 1, except that the gaze cues were replaced with an arrow, measuring $6.0 \times 2.0 \mathrm{~cm}$, presented in the center of the screen. Appearing first as a single line for $1,500 \mathrm{msec}$, two arrow heads appeared $500 \mathrm{msec}$ before target onset, to provide the uninformative directional cue. As before, 24 adults ( 3 of them male) volunteered for this experiment. The mean age was 24.2 years $(S D=4.30)$, and all had normal or corrected-to-normal vision. The participants were recruited from the School of Psychology's community participant panel, gave informed consent, and received payment for participation.

\section{Results}

Arrow cuing. Accuracy in categorizing the targets was $94.4 \%$. Again, there was no effect of cuing on accuracy in the standard blocks $[t(23)=0.343, p=.74]$ or the ratings block $[t(23)=-1.619, p=.12]$. A cuing effect did emerge in the median RT analysis, with RTs being shorter to congruent than to incongruent targets in the standard $[t(23)=3.94, p<.001$, one-tailed $]$ and ratings $[t(23)=$ $1.97, p=.031$, one-tailed] blocks (see Table 1). Further analyses compared the RT effects between the two experiments. The cuing $\times$ experiment interaction was not significant $(F<1)$.

Object ratings. The participants' cuing effects in this experiment were virtually identical to those in Experiment 1 . However, the critical question is whether an arrow cue was just as effective as gaze cues at eliciting a difference in the appraisals of objects that were either congruent or incongruent. As one can see from Figure 2, it is clear that incongruent objects were liked just as much $(M=$ $4.89, S D=0.96$, range $=3.99$ ) as congruent objects $(M=4.88, S D=0.96$, range $=4.35)$, when analyzed across participants $[t(23)=0.32, p=.75]$. Similarly, no effect was found when ratings were analyzed across materials $[t(35)=0.43, p=.67]$.

A further analysis in which the ratings across the two experiments were compared demonstrated that overall ratings across the experiments were not different, since the main effect of experiment was nonsignificant $[F(1,46)<1$, $\left.M S_{\mathrm{e}}=1.61, p=.41\right]$. However, most important, the experiment $\times$ cuing interaction was significant $[F(1,46)=$ $\left.7.09, M S_{\mathrm{e}}=0.023, p=.011\right]$, confirming the differential influence of the two cue types on liking.

\section{Discussion}

The results of this second experiment are clear: In the case of cuing via arrows, congruent items are not evaluated higher on a liking scale than incongruent items. Since both gaze (Experiment 1) and arrow cues (Experiment 2) produced similar cuing magnitudes, it is fair to say that attention was cued to the same extent in both experiments. The failure to replicate higher ratings for congruent objects in Experiment 2 is, therefore, very striking. ${ }^{1}$ This implies that attentional cuing via a central cue is not sufficient to produce a difference between how much people like congruent and incongruent target objects. Rather, it is observing another person look at an object that leads to a modulation in the affective response to that object.

\section{GENERAL DISCUSSION}

It has been well established that we automatically follow the direction of another person's gaze and that we respond differently to direct and averted gaze when making personality judgments of the gazing person (Bayliss \& Tipper, 2006; Macrae, Hood, Milne, Rowe, \& Mason, 2002). Here, we demonstrated not only that things that people look at are attended to by observers, but also that these objects are liked more by observers. This effect is not due to the automatic allocation of attention to the looked-at object, since an arrow cue, just as effective at cuing attention, failed to result in a similar affective response. This suggests that we use the gaze of others to help us evaluate the potential value of objects in the world. That is, if someone looks toward an object, it is because they like it (Baron-Cohen et al., 1995), but if they look away from it, they do not. So, it would benefit us to take on board this information and use it to guide and influence our own evaluative processes.

Of note in our procedure is that although we presented the stimuli six times before we recorded the liking rating, it is possible that the difference may be present even after the first exposure. Nevertheless, whether repeated or single exposures are sufficient to produce the liking effects, they have clear real-life applications. For example, a core technique of the advertising industry is to repeat exposures to products. People (i.e., actors) are used to sell products by making the products appear more attractive than they otherwise would. It is possible that by ensuring that triadic joint attention episodes are established between the actor, product, and consumer, advertisers could potentially boost the affective response associated with their product. Furthermore, although peripheral cues might produce effects different from those presented here (cf. Fenske, Raymond, Kessler, Westoby, \& Tipper, 2005), it is suggested that cues such as arrows are effective in ensuring attentional orienting but that, unlike gaze cues, they do not guarantee an evaluative consequence. It is important to note also that all the participants were asked on debrief what they felt had influenced their ratings. Their answers never involved the cue, focusing instead on color, familiarity, and usefulness. This indicates that our use of household objects as stimuli prevented the participants from successfully "working out" the purpose of the study. Hence, their ratings were made naively, and thus it appears that the biases shown in Experiment 1 were evoked without the conscious awareness of the participants.

Of course, the effect we present here needs replication and extension. Comparing objects that have high, low, or 
medium levels of preference before the experiment would enable the evaluation of the generality of the effect. It is likely that objects that one is already enthused or repulsed by may be resistant to such manipulations and that only the mundane everyday items we presented can be modulated by gaze. Another issue is that we had only congruent and incongruent trials in this study. Successfully establishing an appropriate neutral condition would allow for evaluation of the relative influence of increased ratings for looked-at objects, as compared with decreased liking of incongruent items. Furthermore, since the participants responded to the items every time they appeared, what effect a gaze cue would have on ratings of objects that are not behaviorally relevant (i.e., in a passive-viewing condition) is an interesting question for further work.

A final issue of note is the role of the face-object pairings in this study. The evaluative conditioning literature demonstrates that pairing a pleasant stimulus with a neutral stimulus can result in higher ratings for the neutral stimuli, in comparison with neutral stimuli paired with unpleasant stimuli (see De Houwer, Thomas, \& Baeyens, 2001 , for a review). In this study, face pleasantness is unlikely to have had an effect, since the face cue was randomly selected on each trial, so systematic differences in the pleasantness of the faces in the cued and the uncued conditions are unlikely to account for this result. However, observing faces activates neural systems regulating affect per se (see Adolphs, 1999, for a review). Perhaps the generally positive experience of face viewing resulted in an activation of affective circuits in Experiment 1, whereas Experiment 2 was devoid of these (generally rewarding) social stimuli. Future work is planned in which the influence of facial attractiveness and emotional expression on these type of effects (gaze-cuing and liking effects) will be systematically investigated. Investigating these issues is clearly very important for future research into this effect.

In conclusion, we have demonstrated, in a gaze-cuing procedure, that people like objects more when they are looked at by another person. This result underlines the importance of other people's gaze behavior in influencing our own behavior. This is, therefore, another demonstration of the flexibility of the person perception systems in guiding our social interactions and the consequences of those interactions.

\section{REFERENCES}

ADOLPHS, R. (1999). Social cognition and the human brain. Trends in Cognitive Sciences, 3, 469-479.

ARgYLE, M., \& CoOK, M. (1976). Gaze and mutual gaze. Cambridge: Cambridge University Press.

Baron-CoHEN, S. (1995). The eye direction detector (EDD) and the shared attention mechanism (SAM): Two cases for evolutionary psychology. In C. Moore \& P. J. Dunham (Eds.), Joint attention: Its origins and role in development (pp. 41-59). Hillsdale, NJ: Erlbaum.

Baron-Cohen, S., Campbell, R., Karmiloff-Smith, A., Grant, J., \& WALKER, J. (1995). Are children with autism blind to the mentalistic significance of the eyes? British Journal of Developmental Psychology, 13, 379-398.

Bayliss, A. P., di Pellegrino, G., \& TipPer, S. P. (2004). Orienting of attention via observed eye gaze is head-centred. Cognition, 94, B1B10.
Bayliss, A. P., di Pellegrino, G., \& Tipper, S. P. (2005). Sex differences in eye gaze and symbolic cueing of attention. Quarterly Journal of Experimental Psychology, 58A, 631-650.

BaYLiss, A. P., \& TIPPER, S. P. (2006). Predictive gaze cues and personality judgments: Should eye trust you? Psychological Science, 17, 514-520.

DAVIDSON, R. J., \& IRWIN, W. (1999). The functional neuroanatomy of emotional affective style. Trends in Cognitive Sciences, 3, 11-21.

De Houwer, J., Thomas, S., \& BaEYens, F. (2001). Associative learning of likes and dislikes: A review of 25 years of research on human evaluative conditioning. Psychological Bulletin, 127, 853-869.

EMERY, N. J. (2000). The eyes have it: The neuroethology, function and evolution of social gaze. Neuroscience \& Biobehavioral Reviews, 24 , 581-604.

Fenske, M., Raymond, J., Kessler, K., Westoby, N., \& TipPer, S. P. (2005). Attentional inhibition has social-emotional consequences for unfamiliar faces. Psychological Science, 16, 753-758.

Friesen, C. K., \& Kingstone, A. (1998). The eyes have it! Reflexive orienting is triggered by nonpredictive gaze. Psychonomic Bulletin \& Review, 5, 490-495.

FrIesEN, C. K., \& KINGSTONE, A. (2003). Abrupt onsets and gaze direction cues trigger independent reflexive attentional effects. Cognition, 87, B1-B10.

FrISCHEN, A., \& ThPPER, S. P. (2004). Orienting attention via observed gaze shift evokes longer term inhibitory effects: Implications for social interactions, attention, and memory. Journal of Experimental Psychology: General, 133, 516-533.

LANGTON, S. R. H., \& BRUCE, V. (1999). Reflexive visual orienting in response to the social attention of others. Visual Cognition, 6, 541567.

Macrae, C. N., Hood, B. M., Milne, A. B., Rowe, A. C., \& Mason, M. F. (2002). Are you looking at me? Eye gaze and person perception. Psychological Science, 13, 460-464.

MOORE, C., \& DUNHAM, P. J. (EDs.) (1995). Joint attention: Its origins and role in development. Hillsdale, NJ: Erlbaum.

REBER, R., Winkielman, P., \& SCHWARZ, N. (1998). Effects of perceptual fluency on affective judgments. Psychological Research, 9, 45-48.

SHEPHERD, M., FindLAY, J. M., \& HoCKeY, R. J. (1986). The relationship between eye movements and spatial attention. Quarterly Journal of Experimental Psychology, 38A, 475-491.

Shimojo, S., Simion, C., Shimojo, E., \& Scheier, C. (2003). Gaze bias both reflects and influences preference. Nature Neuroscience, 6, 1317-1322.

TIPPLES, J. (2002). Eye gaze is not unique: Automatic orienting in response to uninformative arrows. Psychonomic Bulletin \& Review, 9 , 314-318.

VeCERA, S. P., \& Johnson, M. H. (1995). Gaze detection and the cortical processing of faces: Evidence from infants and adults. Visual Cognition, 2, 101-129.

\section{NOTE}

1. An anonymous reviewer suggested that the lack of a ratings effect in Experiment 2 may have been due to the fact that Experiment 1 recruited unpaid psychology undergraduates and Experiment 2 recruited slightly older, paid participants with no knowledge of psychology. We understand the potential influence of such factors in these type of studies. Hence, we recruited 24 age-matched $(M=19.7$ years, $S D=1.4)$ participants from the psychology undergraduate panel and replicated Experiment 2 . The findings were virtually identical to those with the paid participants. Cuing was found in median $\operatorname{RTs}[t(23)=2.99, p=.004$, one-tailed], but not in errors $(p>.18)$. The ratings for congruent objects $(M=4.82, S D=0.55)$ did not differ from those for incongruent objects $(M=4.80, S D=0.51)[t(23)=0.372, p=.71]$, and this null effect interacted significantly with the liking effect in Experiment $1[F(1,46)=$ $\left.4.91, M S_{\mathrm{e}}=0.021, p=.032\right]$; the ratings were not significantly different overall between the experiments $(F<1)$.

(Manuscript received September 13, 2005; revision accepted for publication April 21, 2006.) 